\title{
Approximate Analytical Solutions for Mathematical Model of Tumour Invasion and Metastasis Using Modified Adomian Decomposition and Homotopy Perturbation Methods
}

\author{
Norhasimah Mahiddin' ${ }^{1}$ and S. A. Hashim Ali ${ }^{2}$ \\ ${ }^{1}$ Institute of Mathematical Sciences, University of Malaya, 50603 Kuala Lumpur, Malaysia \\ ${ }^{2}$ Faculty of Science \& Technology, Open University Malaysia, 50603 Kuala Lumpur, Malaysia \\ Correspondence should be addressed to Norhasimah Mahiddin; norhasimah@siswa.um.edu.my
}

Received 21 August 2013; Revised 9 December 2013; Accepted 16 December 2013; Published 30 January 2014

Academic Editor: Hak-Keung Lam

Copyright ( 2014 N. Mahiddin and S. A. Hashim Ali. This is an open access article distributed under the Creative Commons Attribution License, which permits unrestricted use, distribution, and reproduction in any medium, provided the original work is properly cited.

\begin{abstract}
The modified decomposition method (MDM) and homotopy perturbation method (HPM) are applied to obtain the approximate solution of the nonlinear model of tumour invasion and metastasis. The study highlights the significant features of the employed methods and their ability to handle nonlinear partial differential equations. The methods do not need linearization and weak nonlinearity assumptions. Although the main difference between MDM and Adomian decomposition method (ADM) is a slight variation in the definition of the initial condition, modification eliminates massive computation work. The approximate analytical solution obtained by MDM logically contains the solution obtained by HPM. It shows that HPM does not involve the Adomian polynomials when dealing with nonlinear problems.
\end{abstract}

\section{Introduction}

Over the years, many mathematical models of tumour growth have appeared in literature [1-3]. These problems and phenomena are modeled by partial differential equations (PDE) such as deterministic reaction-diffusion equations which are used to model the spatial spread of tumours both at early growth and later invasive stages $[4,5]$. In most cases, these problems do not admit analytical solution. So these equations should be solved using some particular techniques. Chaplain [6] used numerical solution (finite difference method) to solve the above problem. However, this method involved linearization, discretization, and assumption. Therefore, the real problem has to undergo simplification before it can be solved. In recent years, much attention has been devoted to the newly developed methods to construct an analytical solution of equation such as Adomian decomposition method (ADM) [7] and homotopy perturbation method (HPM) [8]. Both methods yield rapidly convergent series solutions for linear and nonlinear equations. The advantages of these methods are that they provide direct scheme for solving the problem, that is, without the need for linearization and discretization. The accuracy of the ADM method was studied extensively by Hashim et al. [9] and compared with other methods [10, 11]. Anderson et al. [12] proposed a modification of the ADM by a slight variation from the standard ADM. The modified method (MDM) was established based on the assumption that the initial function can be divided into two parts and the success of the MDM depends mainly on the proper choice of the parts. In this paper, we present approximate analytical solution of tumour invasion and metastasis model [13] solved by MDM and HPM. The results from both methods are then compared and reveal their capability, effectiveness and convenience. Both methods give successive approximations of high accuracy solution.

\section{Problem Formulation}

Let us consider a system describing the interactions of the tumour cells (denoted by $n$ ), extra cellular matrix (ECM, 
denoted by $f$ ), and matrix degrading enzymes (MDE, denoted by $m$ ) is given by [13]

$$
\begin{gathered}
\frac{\partial n}{\partial t}=D_{n} \nabla^{2} n-\chi \nabla \cdot(n \nabla f), \\
\frac{\partial f}{\partial t}=-\delta m f, \\
\frac{\partial m}{\partial t}=D_{m} \nabla^{2} m+\mu n-\lambda m,
\end{gathered}
$$

where $D_{n}$ is the tumour cell random motility coefficient, $D_{m}$ is the MDE diffusion coefficient, $\chi$ is the haptotactic coefficient, and $\lambda, \mu, \delta$ are the positive constants.

Non-dimensionalise of (1) by setting

$$
\begin{gathered}
\tilde{n}=\frac{n}{n_{o}}, \quad \tilde{f}=\frac{f}{f_{o}}, \quad \tilde{m}=\frac{m}{m_{o}}, \\
\tilde{x}=\frac{x}{L}, \quad \tilde{t}=\frac{t}{\tau},
\end{gathered}
$$

where $n_{o}$ is the tumour cell density, $f_{o}$ is the ECM density, $m_{o}$ is the MDE concentration, $L$ is the length scale, and $\tau$ is the time $\left(\tau=L^{2} / D\right.$, where $D$ is a reference chemical diffusion coefficient). By dropping the tildes for notational convenience, we obtain the scaled system of equations:

$$
\begin{gathered}
\frac{\partial n}{\partial t}=d_{n} \nabla^{2} n-\gamma \nabla \cdot(n \nabla f), \\
\frac{\partial f}{\partial t}=-\eta m f, \\
\frac{\partial m}{\partial t}=d_{m} \nabla^{2} m+\omega n-\beta m,
\end{gathered}
$$

where $d_{n}=D_{n} / D, \gamma=\chi f_{o} / D, \eta=\tau m_{o} \delta, d_{m}=D_{m} / D, \omega=$ $\tau \mu n_{o} / m_{o}$, and $\beta=\tau \lambda$. The initial conditions of each equation are

$$
\begin{gathered}
n(x, 0)=\exp \left(-\frac{x^{2}}{\varepsilon}\right), \\
f(x, 0)=1-0.5 \exp \left(-\frac{x^{2}}{\varepsilon}\right), \\
m(x, 0)=0.5 \exp \left(-\frac{x^{2}}{\varepsilon}\right),
\end{gathered}
$$

where $\varepsilon$ is a positive constant.

The approximate solutions of (3)-(5) are obtained by integrating each equation once with respect to $t$ and using the initial condition. Hence we obtained

$$
\begin{gathered}
n(x, t)=n(x)+d_{n} \int_{0}^{t} \frac{\partial^{2} n}{\partial x^{2}} d z \\
-\gamma \int_{0}^{t} \frac{\partial n}{\partial x} \cdot \frac{\partial f}{\partial x} d z-\gamma \int_{0}^{t} n \frac{\partial^{2} f}{\partial x^{2}} d z \\
f(x, t)=f(x)-\eta \int_{0}^{t} m f d z \\
m(x, t)=m(x)+d_{m} \int_{0}^{t} \frac{\partial^{2} m}{\partial x^{2}} d z+\omega \int_{0}^{t} n d z-\beta \int_{0}^{t} m d z
\end{gathered}
$$

In (7)-(9), we assume $n(x), f(x)$, and $m(x)$ are bounded for all $x$ in $J=[0, T],(T \in \Re)$, and $|t-\tau| \leq m^{\prime}$, for all $0 \leq t$, $\tau \leq T$. The terms $\left(\partial^{2} n / \partial x^{2}\right),(\partial n / \partial x) \cdot(\partial f / \partial x), n\left(\partial^{2} f / \partial x^{2}\right)$, $F_{1}(m f)=m f, \partial^{2} m / \partial x^{2}, F_{2}(n)=n$, and $F_{3}(m)=m$ are Lipschitz continuous with

$$
\begin{gathered}
\left|\frac{\partial^{2} n}{\partial x^{2}}-\frac{\partial^{2} n^{*}}{\partial x^{2}}\right| \leq L_{1}\left|n-n^{*}\right|, \\
\left|\frac{\partial n}{\partial x} \cdot \frac{\partial f}{\partial x}-\frac{\partial n^{*}}{\partial x} \cdot \frac{\partial f^{*}}{\partial x}\right| \leq L_{2}\left|n f-n^{*} f^{*}\right|, \\
\left|n \frac{\partial^{2} f}{\partial x^{2}}-n^{*} \frac{\partial^{2} f^{*}}{\partial x^{2}}\right| \leq L_{3}\left|n f-n^{*} f^{*}\right|, \\
\left|F_{1}(m, f)-F_{1}\left(m^{*}, f^{*}\right)\right| \leq L_{4}\left|m f-m f^{*}\right|, \\
\left|\frac{\partial^{2} m}{\partial x^{2}}-\frac{\partial^{2} m^{*}}{\partial x^{2}}\right| \leq L_{5}\left|m-m^{*}\right|, \\
\left|F_{2}(n)-F_{2}\left(n^{*}\right)\right| \leq L_{6}\left|n-n^{*}\right|, \\
\left|F_{3}(m)-F_{3}\left(m^{*}\right)\right| \leq L_{7}\left|m-m^{*}\right|, \\
\alpha=T\left(m^{\prime} L_{1}+m^{\prime} L_{2}+m^{\prime} L_{3}+m^{\prime} L_{4}\right. \\
\left.+m^{\prime} L_{5}+m^{\prime} L_{6}+m^{\prime} L_{7}\right), \\
\zeta=1-T(1-\alpha) .
\end{gathered}
$$

\section{Mathematical Methods}

3.1. Adomian Decomposition Method (ADM). The Adomian decomposition method is applied in (3)-(5):

$$
\begin{gathered}
L_{t} n=d_{n} \frac{\partial^{2} n}{\partial x^{2}}-\gamma\left[\frac{\partial n}{\partial x} \frac{\partial f}{\partial x}+n \frac{\partial^{2} f}{\partial x^{2}}\right], \\
L_{t} f=-\eta m f, \\
L_{t} m=d_{m} \frac{\partial^{2} m}{\partial x^{2}}+\omega n-\beta m,
\end{gathered}
$$

where $L_{t}=\partial / \partial t$ is integrable differential operator with $L_{t}^{-1}=$ $\int_{0}^{t}(\cdot) d t$.

Operating on both sides of (11)-(13) with the integral operator $L^{-1}$ leads to

$$
\begin{gathered}
n(x, t)=n(x, 0)+d_{n} L_{t}^{-1}\left(\frac{\partial^{2} n}{\partial x^{2}}\right) \\
-\gamma\left[L_{t}^{-1}\left[N_{1}(n, f)\right]+L_{t}^{-1}\left[N_{2}(n, f)\right]\right], \\
f(x, t)=f(x, 0)-\eta L_{t}^{-1}\left[N_{3}(m, f)\right], \\
m(x, t)=m(x, 0)+L_{t}^{-1}\left(d_{m} \frac{\partial^{2} m}{\partial x^{2}}\right)+L_{t}^{-1}[\omega n-\beta m],
\end{gathered}
$$


where

$$
\begin{gathered}
N_{1}(n, f)=\frac{\partial n}{\partial x} \frac{\partial f}{\partial x} \\
N_{2}(n, f)=n \frac{\partial^{2} f}{\partial x^{2}} \\
N_{3}(m, f)=m f
\end{gathered}
$$

are the nonlinear terms. The solutions $n(x, t), f(x, t)$, and $m(x, t)$ can be decomposed by an infinite series as follows [7]:

$$
\begin{aligned}
n(x, t) & =\sum_{i=0}^{\infty} n_{i}(x, t), \\
f(x, t) & =\sum_{i=0}^{\infty} f_{i}(x, t), \\
m(x, t) & =\sum_{i=0}^{\infty} m_{i}(x, t),
\end{aligned}
$$

where $n_{i}(x, t), f_{i}(x, t)$, and $m_{i}(x, t)$ are the components of $n(x, t), f(x, t)$, and $m(x, t)$ that will elegantly be determined. The nonlinear term $N(x, t)$ is decomposed by the following infinite series:

$$
N_{k}(x, t)=\sum_{l=0}^{\infty} A_{k l}, \quad k=1,2,3
$$

where $A_{k l}$ is called Adomian's polynomial and defined by

$$
\begin{array}{r}
A_{k l}=\frac{1}{l !}\left[\frac{d^{l}}{d \psi^{l}} N_{k}\left(\sum_{i=0}^{\infty} \psi^{i} n_{i}, \sum_{i=0}^{\infty} \psi^{i} f_{i}, \sum_{i=0}^{\infty} \psi^{i} m_{i}\right)\right]_{\psi=0}, \\
i \geq 0 .
\end{array}
$$

From the above consideration, the decomposition method defines the components $n_{i}(x, t), f_{i}(x, t)$, and $m_{i}(x, t)$ for $i \geq 0$ by the following recursive relationships.

Anderson et al. [12] proposed that the construction of the zeroth component of the decomposition series can be defined in a slightly different way. The modified method (MDM) was established based on the assumption that if the zeroth component $u_{o}=g$ and the function $g$ is possible to divide into two parts such as $g_{1}$ and $g_{2}$, one can formulate the recursive algorithm for $u_{o}$ and general term $u_{n+1}$ in a form of the modified recursive scheme as follows:

for $n_{i}(x, t)$,

$$
\begin{gathered}
n_{0}=g_{1}, \\
n_{1}=g_{2}+\int_{0}^{t}\left[d_{n} \frac{\partial^{2} n_{0}}{\partial x^{2}}-\gamma\left\{A_{1,0}(n, f)+A_{2,0}(n, f)\right\}\right] d \tau \\
n_{l+1}(x, t)=\int_{0}^{t}\left[d_{n} \frac{\partial^{2} n_{l}}{\partial x^{2}}-\gamma\left\{A_{1, l}(n, f)+A_{2, l}(n, f)\right\}\right] d \tau,
\end{gathered}
$$

for $f_{i}(x, t)$,

$$
\begin{gathered}
f_{0}=g_{1}^{\prime}, \\
f_{1}=g_{2}^{\prime}-\eta \int_{0}^{t}\left[A_{3,0}(m, f)\right] d \tau, \\
f_{l+1}(x, t)=-\eta \int_{0}^{t}\left[A_{3, l}(m, f)\right] d \tau, \quad l \geq 1 ;
\end{gathered}
$$

for $m_{i}(x, t)$,

$$
\begin{gathered}
m_{0}=g_{1}^{\prime \prime}, \\
m_{1}=g_{2}^{\prime \prime}+\int_{0}^{t}\left[d_{m} \frac{\partial^{2} m_{l}}{\partial x^{2}}+\omega n_{l}-\beta m_{l}\right] d \tau \\
m_{l+1}(x, t)=\int_{0}^{t}\left[d_{m} \frac{\partial^{2} m_{l}}{\partial x^{2}}+\omega n_{l}-\beta m_{l}\right] d \tau, \quad l \geq 1 .
\end{gathered}
$$

This type of modification is giving more flexibility to the $\mathrm{ADM}$ in order to solve complicated nonlinear differential equations. MDM scheme avoids the unnecessary computation especially in calculation of the Adomian polynomials. The computation of these polynomials will be reduced very considerably by using the MDM.

3.2. Homotopy Perturbation Method (HPM). To solve (3)(5) with the HPM method, we construct the following homotopy:

$$
\begin{aligned}
H_{1}(n, f, p)= & (1-p)\left(\frac{\partial n}{\partial t}-\frac{\partial n_{0}}{\partial t}\right) \\
& +p\left(\frac{\partial n}{\partial t}-d_{n} \frac{\partial^{2} n}{\partial x^{2}}+\gamma \frac{\partial n}{\partial x} \cdot \frac{\partial f}{\partial x}+\gamma n \frac{\partial^{2} f}{\partial x^{2}}\right) \\
= & 0,
\end{aligned}
$$

$$
H_{2}(f, m, p)=(1-p)\left(\frac{\partial f}{\partial t}-\frac{\partial f_{0}}{\partial t}\right)+p\left(\frac{\partial f}{\partial t}+\eta m f\right)=0
$$

$$
\begin{aligned}
H_{3}(m, n, p)= & (1-p)\left(\frac{\partial m}{\partial t}-\frac{\partial m_{0}}{\partial t}\right) \\
& +p\left(\frac{\partial m}{\partial t}-d_{m} \frac{\partial^{2} m}{\partial x^{2}}-\omega n+\beta m\right)=0
\end{aligned}
$$

or

$$
\begin{aligned}
H_{1}(n, f, p)= & \frac{\partial n}{\partial t}-\frac{\partial n_{0}}{\partial t} \\
& +p\left(-d_{n} \frac{\partial^{2} n}{\partial x^{2}}+\gamma \frac{\partial n}{\partial x} \cdot \frac{\partial f}{\partial x}+\gamma n \frac{\partial^{2} f}{\partial x^{2}}+\frac{\partial n_{o}}{\partial t}\right) \\
= & 0, \\
H_{2}(f, m, p)= & \frac{\partial f}{\partial t}-\frac{\partial f_{0}}{\partial t}+p\left(\eta m f+\frac{\partial f_{0}}{\partial t}\right)=0 \\
H_{3}(m, n, p)= & \frac{\partial m}{\partial t}-\frac{\partial m_{0}}{\partial t} \\
& +p\left(-d_{m} \frac{\partial^{2} m}{\partial x^{2}}-\omega n+\beta m+\frac{\partial m_{0}}{\partial t}\right)=0
\end{aligned}
$$


In HPM, the solutions of (25)-(28) are expressed as power series in $p$ :

$$
\begin{aligned}
n(x, t)= & n_{o}(x, t)+p n_{1}(x, t)+p^{2} n_{2}(x, t) \\
& +p^{3} n_{3}(x, t)+\cdots, \\
f(x, t)= & f_{o}(x, t)+p f_{1}(x, t)+p^{2} f_{2}(x, t) \\
& +p^{3} f_{3}(x, t)+\cdots, \\
m(x, t)= & m_{o}(x, t)+p m_{1}(x, t)+p^{2} m_{2}(x, t) \\
& +p^{3} m_{3}(x, t)+\cdots,
\end{aligned}
$$

where $p \in[0,1]$ is an embedding parameter and $n_{o}, f_{o}$, and $m_{o}$ are the arbitrary initial approximation satisfying the given initial condition. As $p$ approaches to 1, we obtained

$$
\begin{gathered}
n(x, t)=\lim _{p \rightarrow 1} n=n_{0}+n_{1}+n_{2}+n_{3}+\cdots=\sum_{i=0}^{\infty} n_{i}, \\
f(x, t)=\lim _{p \rightarrow 1} f=f_{0}+f_{1}+f_{2}+f_{3}+\cdots=\sum_{i=0}^{\infty} f_{i}, \\
m(x, t)=\lim _{p \rightarrow 1} m=m_{0}+m_{1}+m_{2}+m_{3}+\cdots=\sum_{i=0}^{\infty} m_{i} .
\end{gathered}
$$

Substituting (29)-(31) into (25),

$$
\begin{aligned}
\frac{\partial}{\partial t}\left(n_{0}+p n_{1}+p^{2} n_{2}+p^{3} n_{3}+\cdots\right)-\frac{\partial n_{0}}{\partial t} \\
+p\left[-d_{n} \frac{\partial^{2}}{\partial x^{2}}\left(n_{0}+p n_{1}+p^{2} n_{2}+p^{3} n_{3}+\cdots\right)\right. \\
+\gamma \frac{\partial}{\partial x}\left(n_{0}+p n_{1}+p^{2} n_{2}+p^{3} n_{3}+\cdots\right) \\
\times \frac{\partial}{\partial x}\left(f_{0}+p f_{1}+p^{2} f_{2}+p^{3} f_{3}+\cdots\right) \\
+\gamma\left(n_{0}+p n_{1}+p^{2} n_{2}+p^{3} n_{3}+\cdots\right) \\
\times \frac{\partial^{2}}{\partial x^{2}}\left(f_{0}+p f_{1}+p^{2} f_{2}+p^{3} f_{3}+\cdots\right) \\
\left.+\frac{\partial n_{0}}{\partial t}\right]=0 .
\end{aligned}
$$

Substituting (30)-(31) into (26),

$$
\begin{aligned}
\frac{\partial}{\partial t}\left(f_{0}\right. & \left.+p f_{1}+p^{2} f_{2}+p^{3} f_{3}+\cdots\right)-\frac{\partial f_{0}}{\partial t} \\
+p[ & \eta\left(m_{0}+p m_{1}+p^{2} m_{2}+p^{3} m_{3}+\cdots\right) \\
& \left.\times\left(f_{0}+p f_{1}+p^{2} f_{2}+p^{3} f_{3}+\cdots\right)+\frac{\partial f_{0}}{\partial t}\right]=0 .
\end{aligned}
$$

Substituting (29)-(31) into (27),

$$
\begin{gathered}
\frac{\partial}{\partial t}\left(m_{0}+p m_{1}+p^{2} m_{2}+p^{3} m_{3}+\cdots\right)-\frac{\partial m_{0}}{\partial t} \\
+p\left[-d_{m} \frac{\partial^{2}}{\partial x^{2}}\left(m_{0}+p m_{1}+p^{2} m_{2}+p^{3} m_{3}+\cdots\right)\right. \\
-\alpha\left(n_{0}+p n_{1}+p^{2} n_{2}+p^{3} n_{3}+\cdots\right) \\
+\beta\left(m_{0}+p m_{1}+p^{2} m_{2}+p^{3} m_{3}+\cdots\right) \\
\left.+\frac{\partial m_{0}}{\partial t}\right]=0 .
\end{gathered}
$$

Equating the coefficients of the terms in (32)-(34) with the identical powers of $p$, we obtained the following.

From (32),

$$
\begin{aligned}
& p^{o}: \frac{\partial n_{0}}{\partial t}-\frac{\partial n_{0}}{\partial t}=0, \\
& p^{1}: \frac{\partial n_{1}}{\partial t}-d_{n} \frac{\partial^{2} n_{0}}{\partial x^{2}}+\gamma \frac{\partial n_{0}}{\partial x} \frac{\partial f_{0}}{\partial x}+\gamma n_{0} \frac{\partial^{2} f_{0}}{\partial x^{2}}+\frac{\partial n_{0}}{\partial t}=0, \\
& p^{2}: \frac{\partial n_{2}}{\partial t}-d_{n} \frac{\partial^{2} n_{1}}{\partial x^{2}}+\gamma \frac{\partial n_{0}}{\partial x} \frac{\partial f_{1}}{\partial x}+\gamma \frac{\partial n_{1}}{\partial x} \frac{\partial f_{0}}{\partial x} \\
&+\gamma n_{0} \frac{\partial^{2} f_{1}}{\partial x^{2}}+\gamma n_{1} \frac{\partial^{2} f_{0}}{\partial x^{2}}=0, \\
& p^{3}: \frac{\partial n_{3}}{\partial t}-d_{n} \frac{\partial^{2} n_{2}}{\partial x^{2}}+\gamma \frac{\partial n_{0}}{\partial x} \frac{\partial f_{2}}{\partial x}+\gamma \frac{\partial n_{1}}{\partial x} \frac{\partial f_{1}}{\partial x} \\
&+\gamma \frac{\partial n_{2}}{\partial x} \frac{\partial f_{0}}{\partial x}+\gamma n_{0} \frac{\partial^{2} f_{2}}{\partial x^{2}} \\
&+\gamma n_{1} \frac{\partial^{2} f_{1}}{\partial x^{2}}+\gamma n_{2} \frac{\partial^{2} f_{0}}{\partial x^{2}}=0, \\
&+\gamma \frac{\partial n_{3}}{\partial x} \frac{\partial f_{0}}{\partial x}+\gamma n_{0} \frac{\partial^{2} f_{3}}{\partial x^{2}}+\gamma n_{1} \frac{\partial^{2} f_{2}}{\partial x^{2}}+\gamma n_{2} \frac{\partial^{2} f_{1}}{\partial x^{2}}=0 . \\
& p^{4}: \frac{\partial n_{4}}{\partial t}-d_{n} \frac{\partial^{2} n_{3}}{\partial x^{2}}+\gamma \frac{\partial n_{0}}{\partial x} \frac{\partial f_{3}}{\partial x}+\gamma \frac{\partial n_{1}}{\partial x} \frac{\partial f_{2}}{\partial x}+\gamma \frac{\partial n_{2}}{\partial x} \frac{\partial f_{1}}{\partial x} \\
&+135)
\end{aligned}
$$

From (33),

$$
\begin{aligned}
& p^{o}: \frac{\partial f_{0}}{\partial t}-\frac{\partial f_{0}}{\partial t}=0, \\
& p^{1}: \frac{\partial f_{1}}{\partial t}+\eta m_{0} f_{0}+\frac{\partial f_{0}}{\partial t}=0, \\
& p^{2}: \frac{\partial f_{2}}{\partial t}+\eta m_{0} f_{1}+\eta m_{1} f_{0}=0, \\
& p^{3}: \frac{\partial f_{3}}{\partial t}+\eta m_{0} f_{2}+\eta m_{1} f_{1}+\eta m_{2} f_{0}=0, \\
& p^{4}: \frac{\partial f_{4}}{\partial t}+\eta m_{0} f_{3}+\eta m_{1} f_{2}+\eta m_{2} f_{1}+\eta m_{3} f_{0}=0 .
\end{aligned}
$$


From (34),

$$
\begin{aligned}
& p^{o}: \frac{\partial m_{0}}{\partial t}-\frac{\partial m_{0}}{\partial t}=0 \\
& p^{1}: \frac{\partial m_{1}}{\partial t}-d_{m} \frac{\partial^{2} m_{0}}{\partial x^{2}}-\omega n_{0}+\beta m_{0}+\frac{\partial m_{0}}{\partial t}=0 \\
& p^{2}: \frac{\partial m_{2}}{\partial t}-d_{m} \frac{\partial^{2} m_{1}}{\partial x^{2}}-\omega n_{1}+\beta m_{1}=0, \\
& p^{3}: \frac{\partial m_{3}}{\partial t}-d_{m} \frac{\partial^{2} m_{2}}{\partial x^{2}}-\omega n_{2}+\beta m_{2}=0, \\
& p^{4}: \frac{\partial m_{4}}{\partial t}-d_{m} \frac{\partial^{2} m_{3}}{\partial x^{2}}-\omega n_{3}+\beta m_{3}=0 .
\end{aligned}
$$

\section{Existence and Convergence of MDM and HPM}

Theorem 1. Let $0<\alpha<1$; then (3)-(5) have a unique solution.

Proof. (I) Let $n$ and $n^{*}$ be two different solutions of (7) then

$$
\begin{aligned}
\left|n-n^{*}\right|= & \mid d_{n} \int_{0}^{t}\left[\frac{\partial^{2} n}{\partial x^{2}}-\frac{\partial^{2} n^{*}}{\partial x^{2}}\right] d z \\
& -\gamma \int_{0}^{t}\left(\frac{\partial n}{\partial x} \cdot \frac{\partial f}{\partial x}-\frac{\partial n^{*}}{\partial x} \cdot \frac{\partial f^{*}}{\partial x}\right) d z \\
& \quad-\gamma \int_{0}^{t}\left(n \frac{\partial^{2} f}{\partial x^{2}}-n^{*} \frac{\partial^{2} f^{*}}{\partial x^{2}}\right) d z \mid \\
\leq & \left|d_{n}\right| \int_{0}^{t}\left|\left[\frac{\partial^{2} n}{\partial x^{2}}-\frac{\partial^{2} n^{*}}{\partial x^{2}}\right]\right| d z \\
& +|\gamma| \int_{0}^{t}\left|\frac{\partial n}{\partial x} \cdot \frac{\partial f}{\partial x}-\frac{\partial n^{*}}{\partial x} \cdot \frac{\partial f^{*}}{\partial x}\right| d z \\
& +|\gamma| \int_{0}^{t}\left|n \frac{\partial^{2} f}{\partial x^{2}}-n^{*} \frac{\partial^{2} f^{*}}{\partial x^{2}}\right| d z \\
\leq & T\left(m^{\prime} L_{1}+m^{\prime} L_{2}+m^{\prime} L_{3}\right)\left|n-n^{*}\right|\left|f-f^{*}\right| \\
= & \alpha\left|n-n^{*}\right|,
\end{aligned}
$$

from which we get $(1-\alpha)\left|n-n^{*}\right| \leq 0$. Since $0<\alpha<1$, $\left|n-n^{*}\right|=0$, implies $n=n^{*}$ and completes the proof.

(II) Let $f$ and $f^{*}$ be two different solutions of (8) then

$$
\begin{aligned}
\left|f-f^{*}\right| & =\left|-\eta \int_{0}^{t}\left(F_{1}(m f)-F_{1}\left(m^{*} f^{*}\right)\right) d z\right| \\
& \leq|\eta|\left|\int_{0}^{t}\left(F_{1}(m f)-F_{1}\left(m^{*} f^{*}\right)\right) d z\right| \\
& \leq T\left(m^{\prime} L_{4}\right) \\
& =\alpha\left|f-f^{*}\right|,
\end{aligned}
$$

from which we get $(1-\alpha)\left|f-f^{*}\right| \leq 0$. Since $0<\alpha<1$, $\left|f-f^{*}\right|=0$, implies $f=f^{*}$ and completes the proof.

(III) Let $m$ and $m^{*}$ be two different solutions of (9); then

$$
\begin{aligned}
\left|m-m^{*}\right|= & \mid d_{m} \int_{0}^{t}\left[\frac{\partial^{2} m}{\partial x^{2}}-\frac{\partial^{2} m^{*}}{\partial x^{2}}\right] d z \\
& +\gamma \int_{0}^{t}\left(\left|F_{2}(n)-F_{2}\left(n^{*}\right)\right|\right) d z \\
& \quad-\beta \int_{0}^{t}\left(\left|F_{3}(m)-F_{3}\left(m^{*}\right)\right|\right) d z \mid \\
\leq & \left|d_{m}\right| \int_{0}^{t}\left|\left[\frac{\partial^{2} m}{\partial x^{2}}-\frac{\partial^{2} m^{*}}{\partial x^{2}}\right]\right| d z \\
& +|\alpha| \int_{0}^{t}\left|F_{2}(n)-F_{2}\left(n^{*}\right)\right| d z \\
& +|\beta| \int_{0}^{t}\left|F_{4}(n)-F_{4}\left(n^{*}\right)\right| d z \\
\leq & T\left(m^{\prime} L_{5}+m^{\prime} L_{6}+m^{\prime} L_{7}\right) \\
= & \alpha\left|m-m^{*}\right| ;
\end{aligned}
$$

from which we get $(1-\alpha)\left|m-m^{*}\right| \leq 0$. Since $0<\alpha<1$, $\left|m-m^{*}\right|=0$, implies $m=m^{*}$ and completes the proof.

Theorem 2. The series solution $n(x, t)=\sum_{i=0}^{\infty} n_{i}(x, t)$, $f(x, t)=\sum_{i=0}^{\infty} f_{i}(x, t)$, and $m(x, t)=\sum_{i=0}^{\infty} m_{i}(x, t)$ of $(3)-(5)$, respectively, using $M D M$ converges if $0<\alpha<1,\left|n_{1}(x, t)\right|<\infty$, $\left|f_{1}(x, t)\right|<\infty$, and $\left|m_{1}(x, t)\right|<\infty$.

Proof. Denote by $(C[J],\|\cdot\|)$ the Banach space of all continuous functions on $J$ with the norm $\|f(t)\|=\max |f(t)| \forall_{t} \in J$. Define the sequence of partial series $\left\{S_{b}\right\}$; let $S_{b}$ and $S_{a}$ be arbitrary partial sums with $b \geq a$. We prove that $S_{b}$ is a Cauchy sequence in this Banach space.

(I) For (11),

$$
\begin{aligned}
&\left\|S_{b}-S_{a}\right\|=\max _{\forall t \in J}\left|S_{b}-S_{a}\right| \\
&=\max _{\forall t \in J}\left|\sum_{i=k+1}^{b} n_{i}(x, t)\right| \\
&=\max _{\forall t \in J} \mid \sum_{i=k+1}^{b}\left(\int_{0}^{t} d_{n} \frac{\partial^{2} n_{i}}{\partial x^{2}} d z\right. \\
& \quad-\gamma \int_{0}^{t} \frac{\partial n_{i}}{\partial x} \cdot \frac{\partial f_{i}}{\partial x} d z \\
&\left.\quad-\gamma \int_{0}^{t} n \frac{\partial^{2} f_{i}}{\partial x^{2}} d z\right) \mid
\end{aligned}
$$




$$
\begin{aligned}
=\max _{\forall t \in J} \mid d_{n} & \int_{0}^{t}\left(\sum_{i=k}^{b-1} \frac{\partial^{2} n_{i}}{\partial x^{2}}\right) d z \\
& -\gamma \int_{0}^{t}\left(\sum_{i=k}^{b-1} \frac{\partial n_{i}}{\partial x} \cdot \frac{\partial f_{i}}{\partial x}\right) d z \\
& -\gamma \int_{0}^{t}\left(\sum_{i=k}^{b-1} n_{i} \frac{\partial^{2} f_{i}}{\partial x^{2}}\right) d z \mid
\end{aligned}
$$

From [14], we have

$$
\begin{gathered}
\sum_{i=k}^{b-1} \frac{\partial^{2} n_{i}}{\partial x^{2}}=G_{1}^{2}\left(S_{b-1}\right)-G_{1}^{2}\left(S_{a-1}\right), \\
\sum_{i=k}^{b-1} \frac{\partial n_{i}}{\partial x} \cdot \frac{\partial f_{i}}{\partial x}=G_{2}^{2}\left(S_{b-1}\right)-G_{2}^{2}\left(S_{a-1}\right), \\
\sum_{i=k}^{b-1} n_{i} \frac{\partial^{2} f_{i}}{\partial x^{2}}=G_{3}^{2}\left(S_{b-1}\right)-G_{3}^{2}\left(S_{a-1}\right) .
\end{gathered}
$$

So,

$$
\begin{aligned}
& \left\|S_{b}-S_{a}\right\| \\
& =\max _{\forall t \in J} \mid d_{n} \int_{0}^{t}\left[G_{1}^{2}\left(S_{b-1}\right)-G_{1}^{2}\left(S_{a-1}\right)\right] d z \\
& -\gamma \int_{0}^{t}\left[G_{2}^{2}\left(S_{b-1}\right)-G_{2}^{2}\left(S_{a-1}\right)\right] d z \\
& \leq\left|d_{n}\right| \int_{0}^{t}\left|G_{1}^{2}\left(S_{b-1}\right)-G_{1}^{2}\left(S_{a-1}\right)\right| d z \\
& +|\gamma| \int_{0}^{t}\left|G_{2}^{2}\left(S_{b-1}\right)-G_{2}^{2}\left(S_{a-1}\right)\right| d z \\
& +|\gamma| \int_{0}^{t}\left|G_{3}^{2}\left(S_{b-1}\right)-G_{3}^{2}\left(S_{a-1}\right)\right| d z \\
& \leq \alpha\left\|S_{b}-S_{a}\right\| .
\end{aligned}
$$

(II) For (12),

$$
\begin{aligned}
\left\|S_{b}-S_{a}\right\| & =\max _{\forall t \in J}\left|S_{b}-S_{a}\right| \\
& =\max _{\forall t \in J}\left|\sum_{i=k+1}^{b} f_{i}(x, t)\right| \\
& =\max _{\forall t \in J}\left|\sum_{i=k+1}^{b}\left(-\eta \int_{0}^{t} m_{i} f_{i} d z\right)\right| \\
& =\max _{\forall t \in J}\left|-\eta \int_{0}^{t}\left(\sum_{i=k}^{b-1} m_{i} f_{i}\right) d z\right| .
\end{aligned}
$$

From [14], we have

$$
\sum_{i=k}^{b-1} m_{i} f_{i}=F_{1}\left(S_{b-1}\right)-F_{1}\left(S_{a-1}\right) .
$$

So,

$$
\begin{aligned}
& \begin{aligned}
\left\|S_{b}-S_{a}\right\| & =\max _{\forall t \in J}\left|-\eta \int_{0}^{t}\left[F_{1}\left(S_{b-1}\right)-F_{1}\left(S_{a-1}\right)\right] d z\right| \\
& \leq|\eta| \int_{0}^{t}\left|F_{1}\left(S_{b-1}\right)-F_{1}\left(S_{a-1}\right)\right| d z \\
& \leq \alpha\left\|S_{b}-S_{a}\right\|
\end{aligned} \\
& \text { (III) For (13) }
\end{aligned}
$$

$$
\begin{aligned}
\left\|S_{b}-S_{a}\right\| & =\max _{\forall t \in J}\left|S_{b}-S_{a}\right| \\
& =\max _{\forall t \in J}\left|\sum_{i=k+1}^{b} m_{i}(x, t)\right| \\
& =\max _{\forall t \in J} \mid \sum_{i=k+1}^{b}\left(\int_{0}^{t} d_{m} \frac{\partial^{2} m_{i}}{\partial x^{2}} d z+\omega \int_{0}^{t} n_{i} d z\right. \\
& =\max _{\forall t \in J} \mid d_{m} \int_{0}^{t}\left(\sum_{i=k}^{b-1} \frac{\partial^{2} m_{i}}{\partial x^{2}}\right) d z+\omega \int_{0}^{t}\left(\sum_{i=k}^{b-1} n_{i}\right) d z \\
& \left.-\beta \int_{0}^{t}\left(\sum_{i=k}^{b-1} m_{i}\right) d z\right) \mid .
\end{aligned}
$$

From [14], we have

$$
\begin{gathered}
\sum_{i=k}^{b-1} \frac{\partial^{2} m_{i}}{\partial x^{2}}=G_{4}^{2}\left(S_{b-1}\right)-G_{4}^{2}\left(S_{a-1}\right), \\
\sum_{i=k}^{b-1} n_{i}=F_{2}\left(S_{b-1}\right)-F_{2}\left(S_{a-1}\right), \\
\sum_{i=k}^{b-1} m_{i}=F_{3}\left(S_{b-1}\right)-F_{3}\left(S_{a-1}\right) .
\end{gathered}
$$


So,

$$
\begin{aligned}
& \left\|S_{b}-S_{a}\right\| \\
& =\max _{\forall t \in J} \mid d_{m} \int_{0}^{t}\left[G_{4}^{2}\left(S_{b-1}\right)-G_{4}^{2}\left(S_{a-1}\right)\right] d z \\
& +\omega \int_{0}^{t}\left[F_{2}\left(S_{b-1}\right)-F_{2}\left(S_{a-1}\right)\right] d z \\
& \quad-\beta \int_{0}^{t}\left[F_{3}\left(S_{b-1}\right)-F_{3}\left(S_{a-1}\right)\right] d z \mid \\
& \leq\left|d_{m}\right| \int_{0}^{t}\left|G_{4}^{2}\left(S_{b-1}\right)-G_{4}^{2}\left(S_{a-1}\right)\right| d z \\
& +|\omega| \int_{0}^{t}\left|F_{2}\left(S_{b-1}\right)-F_{2}\left(S_{a-1}\right)\right| d z \\
& \quad+|\beta| \int_{0}^{t}\left|F_{3}\left(S_{b-1}\right)-F_{3}\left(S_{a-1}\right)\right| d z \\
& \leq \alpha\left\|S_{b}-S_{a}\right\| .
\end{aligned}
$$

For (43), let $b=a+1$; then

$$
\begin{aligned}
\left\|S_{a+1}-S_{a}\right\| \leq & \alpha\left\|S_{a}-S_{a-1}\right\| \\
\leq & \alpha^{2}\left\|S_{a-1}-S_{a-2}\right\| \\
& \vdots \\
\leq & \alpha^{a}\left\|S_{1}-S_{0}\right\| .
\end{aligned}
$$

From the triangle inequality, we have

$$
\begin{aligned}
\| S_{b}- & S_{a} \| \\
& \leq\left\|S_{a+1}-S_{a}\right\|+\left\|S_{a+2}-S_{a+1}\right\|+\cdots+\left\|S_{b}-S_{b-1}\right\| \\
& \leq\left(\alpha^{a}+\alpha^{a+1}+\cdots+\alpha^{b-a-1}\right)\left\|S_{1}-S_{0}\right\| \\
& \leq \alpha^{a}\left(1+\alpha+\alpha^{2}+\cdots+\alpha^{b-a-1}\right)\left\|S_{1}-S_{0}\right\| \\
& \leq \alpha^{a}\left(\frac{1-\alpha^{b-a}}{1-\alpha}\right)\left\|n_{1}(x, t)\right\| ;
\end{aligned}
$$

similar steps for (46)

$$
\leq \alpha^{a}\left(\frac{1-\alpha^{b-a}}{1-\alpha}\right)\left\|f_{1}(x, t)\right\|
$$

similar steps for (49)

$$
\leq \alpha^{a}\left(\frac{1-\alpha^{b-a}}{1-\alpha}\right)\left\|m_{1}(x, t)\right\| .
$$

Since $0<\alpha<1$, we have $\left(1-\alpha^{b-a}\right)<1$; then

$$
\begin{aligned}
\left\|S_{b}-S_{a}\right\| & \leq \frac{\alpha^{a}}{1-\alpha} \max _{\forall t \in J}\left|n_{1}(x, t)\right|, \\
\left\|S_{b}-S_{a}\right\| & \leq \frac{\alpha^{a}}{1-\alpha} \max _{\forall t \in J}\left|f_{1}(x, t)\right|, \\
\left\|S_{b}-S_{a}\right\| & \leq \frac{\alpha^{a}}{1-\alpha} \max _{\forall t \in J}\left|m_{1}(x, t)\right| .
\end{aligned}
$$

But $\left|n_{1}(x, t), f_{1}(x, t), m_{1}(x, t)\right|<\infty$, so as $a \rightarrow \infty$ then $\| S_{b}-$ $S_{a} \| \rightarrow 0$. We confide that $\left\{S_{b}\right\}$ is a Cauchy sequence in $C[J]$; therefore the series converges and the proof is completed.

Theorem 3. If $\left|n_{a}(x, t)\right| \leq 1,\left|f_{a}(x, t)\right| \leq 1,\left|m_{a}(x, t)\right| \leq$ 1 , then the series solution $n(x, t)=\sum_{i=0}^{\infty} n_{i}(x, t), f(x, t)=$ $\sum_{i=0}^{\infty} f_{i}(x, t)$, and $m(x, t)=\sum_{i=0}^{\infty} m_{i}(x, t)$ of $(3)-(5)$ converges to the exact solution by using HPM.

Proof. (I) For (3), we set [14]

$$
\begin{gathered}
\phi_{b}(x, t)=\sum_{i=1}^{b} n_{i}(x, t), \\
\phi_{b+1}(x, t)=\sum_{i=1}^{b+1} n_{i}(x, t) .
\end{gathered}
$$

So,

$$
\begin{aligned}
& \left|\phi_{b+1}(x, t)-\phi_{b}(x, t)\right| \\
& =\left|\phi_{b}+n_{b}-\phi_{b}\right| \\
& =\left|n_{b}\right| \\
& \leq \sum_{k=0}^{a-1}\left(\left|d_{n}\right| \int_{0}^{t}\left|\frac{\partial^{2} n_{a-k-1}}{\partial x^{2}}\right| d z\right. \\
& \quad+|\gamma| \int_{0}^{t}\left|\frac{\partial n_{k-a-1}}{\partial x} \cdot \frac{\partial f_{k-a-1}}{\partial x}\right| d z \\
& \left.+|\gamma| \int_{0}^{t}\left|n_{k-a-1} \frac{\partial^{2} f_{k-a-1}}{\partial x^{2}}\right|\right) d z .
\end{aligned}
$$

Thus

$$
\sum_{b=0}^{\infty}\left\|\phi_{b+1}(x, t)-\phi_{b}(x)\right\| \leq(a-1) \alpha|f(x)| \sum_{b=0}^{\infty} \alpha^{b} .
$$

Since $0<\alpha<1, \lim _{b \rightarrow \infty} n_{b}(x, t)=n(x, t)$.

(II) For (4), we set [14],

$$
\begin{gathered}
\phi_{b}(x, t)=\sum_{i=1}^{b} f_{i}(x, t), \\
\phi_{b+1}(x, t)=\sum_{i=1}^{b+1} f_{i}(x, t) .
\end{gathered}
$$


So,

$$
\begin{aligned}
\left|\phi_{b+1}(x, t)-\phi_{b}(x, t)\right| & =\left|\phi_{b}+f_{b}-\phi_{b}\right| \\
& =\left|f_{b}\right| \\
& \leq \sum_{k=0}^{a-1}|\eta| \int_{0}^{t}\left|m_{k-a-1} f_{k-a-1}\right| d z .
\end{aligned}
$$

Thus

$$
\sum_{b=0}^{\infty}\left\|\phi_{b+1}(x, t)-\phi_{b}(x)\right\| \leq(a-1) \alpha|f(x)| \sum_{b=0}^{\infty} \alpha^{b} .
$$

Since $0<\alpha<1, \lim _{b \rightarrow \infty} f_{b}(x, t)=f(x, t)$.

(III) For (5), we set [14],

$$
\begin{gathered}
\phi_{b}(x, t)=\sum_{i=1}^{b} m_{i}(x, t), \\
\phi_{b+1}(x, t)=\sum_{i=1}^{b+1} m_{i}(x, t) .
\end{gathered}
$$

So,

$$
\begin{aligned}
& \left|\phi_{b+1}(x, t)-\phi_{b}(x, t)\right| \\
& =\left|\phi_{b}+m_{b}-\phi_{b}\right| \\
& =\left|m_{b}\right| \\
& \leq \sum_{k=0}^{a-1}\left(\left|d_{m}\right| \int_{0}^{t}\left|\frac{\partial^{2} m_{a-k-1}}{\partial x^{2}}\right| d z\right. \\
& \quad+|\omega| \int_{0}^{t}\left|n_{k-a-1}\right| d z \\
& \left.\quad+|\beta| \int_{0}^{t}\left|m_{k-a-1}\right|\right) d z .
\end{aligned}
$$

Thus

$$
\sum_{b=0}^{\infty}\left\|\phi_{b+1}(x, t)-\phi_{b}(x)\right\| \leq(a-1) \alpha|f(x)| \sum_{b=0}^{\infty} \alpha^{b} .
$$

Since $0<\alpha<1, \lim _{b \rightarrow \infty} m_{b}(x, t)=m(x, t)$.

\section{Numerical Experiment}

In this section, we compute numerically (3)-(5) by the MDM and HPM methods.

5.1. MDM. From the ADM formula (18), we can obtain the first three terms of the Adomian polynomials:

$$
\begin{aligned}
A_{1,0} & =N_{1}\left(n_{0}, f_{0}\right) \\
& =-\frac{2 x^{2}}{\varepsilon^{2}} e^{-2 x^{2} / \varepsilon},
\end{aligned}
$$

$$
\begin{aligned}
A_{2,0} & =N_{2}\left(n_{0}, f_{0}\right) \\
& =\frac{e^{-2 x^{2} / \varepsilon}}{\varepsilon}\left(1-\frac{2 x^{2}}{\varepsilon}\right), \\
A_{3,0} & =N_{3}\left(m_{0}, f_{0}\right) \\
& =\frac{1}{2} e^{-x^{2} / \varepsilon}\left(1-\frac{1}{2} e^{-x^{2} / \varepsilon}\right) .
\end{aligned}
$$

By the recursive formula in (19)-(21), we can obtain directly the components of $n_{i}, f_{i}$, and $m_{i}$.

From (22),

$$
\begin{aligned}
& n_{o}=0 \text {, } \\
& n_{1}=n(x, 0)+\int_{0}^{t}\left[d_{n} \frac{\partial^{2} n_{0}}{\partial x^{2}}-\gamma\left(A_{10}+A_{20}\right)\right] d \tau \\
& =e^{-x^{2} / \varepsilon}-\left\{2 d_{n}\left[1-\frac{2 x^{2}}{\varepsilon}\right]\right. \\
& \left.+\gamma e^{-x^{2} / \varepsilon}\left[1-\frac{4 x^{2}}{\varepsilon}\right]\right\} \frac{t}{\varepsilon} e^{-x^{2} / \varepsilon}, \\
& n_{2}=-\left(\left\{\frac { - 4 d _ { n } } { \varepsilon } \left\langle\frac{d_{n}}{\varepsilon}\left[3-\frac{6 x^{3}}{\varepsilon}-\frac{2}{\varepsilon}\left(3 x^{2}-\frac{2 x^{5}}{\varepsilon}\right)\right]\right.\right.\right. \\
& \left.\left.+e^{-x^{2} / \varepsilon}\left[\gamma\left(3-\frac{8 x^{3}}{\varepsilon}\right)+3 x^{2}-\frac{4 x^{4}}{\varepsilon}\right]\right\rangle\right\} \\
& +e^{-x^{2} / \varepsilon}\left\langle\frac{2}{\varepsilon^{2}} \gamma \mu x^{2}\left[e^{-x^{2} / \varepsilon}-1\right]\right. \\
& +\frac{4 x}{\varepsilon}\left\{\frac{d_{n}}{\varepsilon}\left(3 x-\frac{2 x^{3}}{\varepsilon}\right)\right. \\
& \left.-\gamma e^{-x^{2} / \varepsilon}\left(3 x-\frac{4 x^{3}}{\varepsilon}\right)\right\} \\
& -\gamma \frac{\mu}{\varepsilon}\left[-1+\frac{2 x^{2}}{\varepsilon}\right. \\
& \left.+e^{-x^{2} / \varepsilon}\left(1-\frac{4 x^{2}}{\varepsilon}\right)\right] \\
& -\gamma\left(1-\frac{2 x^{2}}{\varepsilon}\right) \\
& \times\left\{\frac{2 d_{n}}{\varepsilon}\left(1-\frac{2 x^{2}}{\varepsilon}\right)\right. \\
& \left.\left.\left.+\gamma e^{-x^{2} / \varepsilon}\left(1-\frac{4 x^{2}}{\varepsilon}\right)\right\}\right\rangle\right) \\
& \times \frac{t^{2}}{2} e^{-x^{2} / \varepsilon} .
\end{aligned}
$$


From (23),

$$
\begin{gathered}
f_{o}=0, \\
f_{1}=f(x, 0)-\eta \int_{0}^{t} A_{3,0} d \tau \\
=1-\frac{1}{2} e^{-x^{2} / \varepsilon}-\left(1-\frac{1}{2} e^{-x^{2} / \varepsilon}\right) \frac{\eta t}{2} e^{-x^{2} / \varepsilon}, \\
f_{2}=\left(1-\frac{1}{2} e^{-x^{2} / \varepsilon}\right) \\
\times\left[\frac{\eta}{4} e^{-x^{2} / \varepsilon}-\left(d_{m}\left(1-2 x^{2}\right)-\omega+\frac{\beta}{2}\right)\right] \frac{\eta t^{2}}{2} e^{-x^{2} / \varepsilon} .
\end{gathered}
$$

From (24),

$$
\begin{gathered}
m_{o}=0, \\
m_{1}=\int_{0}^{t}\left[d_{m} \frac{\partial^{2} m_{0}}{\partial x^{2}}+\omega n_{0}-\beta m_{0}\right] d \tau \\
=\frac{1}{2} e^{-x^{2} / \varepsilon}-\left[\frac{d_{m}}{\varepsilon}\left(1-\frac{2 x^{2}}{\varepsilon}\right)-\omega+\frac{\beta}{2}\right] t e^{-x^{2} / \varepsilon}, \\
m_{2}=-\left(\left\{d _ { m } \left[-d_{m}\left\langle\frac{2}{\varepsilon}\left(1-\frac{2 x^{2}}{\varepsilon}\right)\right.\right.\right.\right. \\
\left.+4\left(1-\frac{5 x^{2}}{\varepsilon}+\frac{2 x^{4}}{\varepsilon^{2}}\right)\right\rangle \\
\left.+\omega\left\{\frac{2 d_{n}}{\varepsilon}\left(1-\frac{2 x^{2}}{\varepsilon}\right)(2 \omega-\beta)\right]\right\} \\
\left.+\beta\left[d_{m}\left(1-2 x^{2}\right)-\omega+\frac{\beta}{2}\right]\right) \frac{t^{2}}{2} e^{-x^{2} / \varepsilon} .
\end{gathered}
$$

5.2. HPM Method. Following the HPM method, we can obtain the first three terms of the polynomials.

From (35)-(37),

$$
\begin{gathered}
n_{o}=e^{-x^{2} / \varepsilon}, \\
n_{1}=\int_{0}^{t}\left(d_{n} \frac{2}{\varepsilon} e^{-x^{2} / \varepsilon}\left[1-\frac{2 x^{2}}{\varepsilon}\right]+\gamma \frac{2 x^{2}}{\varepsilon^{2}} e^{-x^{2} / \varepsilon} e^{-x^{2} / \varepsilon}\right. \\
\left.-\gamma e^{-x^{2} / \varepsilon} e^{-x^{2} / \varepsilon}\left[1-\frac{2 x^{2}}{\varepsilon}\right]\right) d \tau \\
=-\left\{2 d_{n}\left[1-\frac{2 x^{2}}{\varepsilon}\right]+\gamma e^{-x^{2} / \varepsilon}\left[1-\frac{4 x^{2}}{\varepsilon}\right]\right\} \frac{t}{\varepsilon} e^{-x^{2} / \varepsilon},
\end{gathered}
$$

$$
n_{2}=\int_{0}^{t}\left(d_{n} \frac{4}{\varepsilon} t e^{-x^{2} / \varepsilon}\right.
$$

$$
\begin{aligned}
& \times\left\{\frac{d_{n}}{\varepsilon}\left[3-\frac{6 x^{3}}{\varepsilon}-\frac{2}{\varepsilon}\left(3 x^{2}-\frac{2 x^{5}}{\varepsilon}\right)\right]\right. \\
& \left.\quad-e^{-x^{2} / \varepsilon}\left[\gamma\left(3-\frac{8 x^{3}}{\varepsilon}\right)+3 x^{2}-\frac{4 x^{4}}{\varepsilon}\right]\right\} \\
& -\frac{2}{\varepsilon} \gamma x e^{-x^{2} / \varepsilon} \frac{\mu}{\varepsilon} t x e^{-x^{2} / \varepsilon}\left[-1+e^{-x^{2} / \varepsilon}\right] \\
& -\frac{4}{\varepsilon} t e^{-x^{2} / \varepsilon}\left\{\frac{d_{n}}{\varepsilon}\left(3 x-\frac{2 x^{3}}{\varepsilon}\right)\right.
\end{aligned}
$$$$
\left.-\gamma e^{-x^{2} / \varepsilon}\left(3 x-\frac{4 x^{3}}{\varepsilon}\right)\right\} x e^{-x^{2} / \varepsilon}
$$$$
+\gamma e^{-x^{2} / \varepsilon} \frac{\mu}{\varepsilon} t e^{-x^{2} / \varepsilon}
$$$$
\times\left[-1+\frac{2 x^{2}}{\varepsilon}+e^{-x^{2} / \varepsilon}-\frac{4 x^{2}}{\varepsilon} e^{-x^{2} / \varepsilon}\right]
$$$$
+\gamma t e^{-x^{2} / \varepsilon}\left\{\frac{2 d_{n}}{\varepsilon}\left(1-\frac{2 x^{2}}{\varepsilon}\right)\right.
$$$$
\left.+\gamma e^{-x^{2} / \varepsilon}\left(1-\frac{4 x^{2}}{\varepsilon}\right)\right\} e^{-x^{2} / \varepsilon}
$$$$
\left.\times\left(1-\frac{2 x^{2}}{\varepsilon}\right)\right) d \tau
$$$$
=-\left(\left\{\frac { - 4 d _ { n } } { \varepsilon } \left\langle\frac { d _ { n } } { \varepsilon } \left[3-\frac{6 x^{3}}{\varepsilon}\right.\right.\right.\right.
$$

$$
\begin{gathered}
\left.-\frac{2}{\varepsilon}\left(3 x^{2}-\frac{2 x^{5}}{\varepsilon}\right)\right] \\
\left.\left.+e^{-x^{2} / \varepsilon}\left[\gamma\left(3-\frac{8 x^{3}}{\varepsilon}\right)+3 x^{2}-\frac{4 x^{4}}{\varepsilon}\right]\right\rangle\right\} \\
+e^{-x^{2} / \varepsilon}\left\langle\frac{2}{\varepsilon^{2}} \gamma \mu x^{2}\left[e^{-x^{2} / \varepsilon}-1\right]\right.
\end{gathered}
$$

$$
+\frac{4 x}{\varepsilon}\left\{\frac{d_{n}}{\varepsilon}\left(3 x-\frac{2 x^{3}}{\varepsilon}\right)\right.
$$$$
\left.-\gamma e^{-x^{2} / \varepsilon}\left(3 x-\frac{4 x^{3}}{\varepsilon}\right)\right\}
$$$$
-\gamma \frac{\mu}{\varepsilon}\left[-1+\frac{2 x^{2}}{\varepsilon}+e^{-x^{2} / \varepsilon}\left(1-\frac{4 x^{2}}{\varepsilon}\right)\right]
$$$$
-\gamma\left(1-\frac{2 x^{2}}{\varepsilon}\right)
$$$$
\times\left\{\frac{2 d_{n}}{\varepsilon}\left(1-\frac{2 x^{2}}{\varepsilon}\right)\right.
$$ 


$$
\left.\left.\left.+\gamma e^{-x^{2} / \varepsilon}\left(1-\frac{4 x^{2}}{\varepsilon}\right)\right\}\right\rangle\right)
$$$$
\times \frac{t^{2}}{2} e^{-x^{2} / \varepsilon}
$$

From (43),

$$
\begin{gathered}
f_{o}=1-\frac{1}{2} e^{-x^{2} / \varepsilon}, \\
f_{1}=-\int_{0}^{t}\left(\frac{\eta}{2} e^{-x^{2} / \varepsilon}\left(1-\frac{1}{2} e^{-x^{2} / \varepsilon}\right)\right) d \tau \\
=-\left(1-\frac{1}{2} e^{-x^{2} / \varepsilon}\right) \frac{\eta t}{2} e^{-x^{2} / \varepsilon} \\
f_{2}=\int_{0}^{t}\left(\eta \left[\frac{1}{2} e^{-x^{2} / \varepsilon} \frac{\eta}{2} e^{-x^{2} / \varepsilon}\left(1-\frac{1}{2} e^{-x^{2} / \varepsilon}\right) t\right.\right. \\
+e^{-x^{2} / \varepsilon}\left(d_{m}\left(1-2 x^{2}\right)-\omega+\frac{\beta}{2}\right) t \\
=\left(1-\frac{1}{2} e^{-x^{2} / \varepsilon}\right) \\
\left.\left.\left.\times\left[\frac{\eta}{4} e^{-x^{2} / \varepsilon}-\left(d_{m}\left(1-2 x^{2}\right)-\omega+\frac{\beta}{2}\right)\right] \frac{\eta t^{2}}{2} e^{-x^{2} / \varepsilon}\right)\right]\right) d \tau
\end{gathered}
$$

From (46),

$$
\begin{gathered}
m_{o}=\frac{1}{2} e^{-x^{2} / \varepsilon}, \\
m_{1}=-\int_{0}^{t} e^{-x^{2} / \varepsilon}\left[\frac{d_{m}}{\varepsilon}\left(1-\frac{2 x^{2}}{\varepsilon}\right)-\omega+\frac{\beta}{2}\right] d \tau \\
=-\left[\frac{d_{m}}{\varepsilon}\left(1-\frac{2 x^{2}}{\varepsilon}\right)-\omega+\frac{\beta}{2}\right] t e^{-x^{2} / \varepsilon}, \\
m_{2}=\int_{0}^{t}\left(-d_{m} t e^{-x^{2} / \varepsilon}\right. \\
\times\left\{-d_{m}\left\langle\frac{2}{\varepsilon}\left(1-\frac{2 x^{2}}{\varepsilon}\right)\right.\right. \\
\left.+\frac{1}{\varepsilon}\left(1-\frac{2 x^{2}}{\varepsilon}\right)(2 \omega-\beta)\right\} \\
-\omega t e^{-x^{2} / \varepsilon}
\end{gathered}
$$

$$
\begin{aligned}
& \times\left\{\frac{2 d_{n}}{\varepsilon}\left(1-\frac{2 x^{2}}{\varepsilon}\right)+\gamma e^{-x^{2} / \varepsilon}\left(1-\frac{4 x^{2}}{\varepsilon}\right)\right\} \\
&+\left.\beta e^{-x^{2} / \varepsilon} t\left[d_{m}\left(1-2 x^{2}\right)-\omega+\frac{\beta}{2}\right]\right) d \tau \\
& m_{2}=-\left(\left\{d _ { m } \left[-d_{m}\left\langle\frac{2}{\varepsilon}\left(1-\frac{2 x^{2}}{\varepsilon}\right)\right.\right.\right.\right. \\
&\left.+4\left(1-\frac{5 x^{2}}{\varepsilon}+\frac{2 x^{4}}{\varepsilon^{2}}\right)\right\rangle\left.\left.+\frac{1}{\varepsilon}\left(1-\frac{2 x^{2}}{\varepsilon}\right)(2 \omega-\beta)\right]\right\} \\
&+\omega\left\{\frac{2 d_{n}}{\varepsilon}\left(1-\frac{2 x^{2}}{\varepsilon}\right)\right. \\
&\left.\times \frac{t^{2}}{2} e^{-x^{2} / \varepsilon} \cdot \quad+\gamma e^{-x^{2} / \varepsilon}\left(1-\frac{4 x^{2}}{\varepsilon}\right)\right\} \\
&\left.-\beta\left[d_{m}\left(1-2 x^{2}\right)-\omega+\frac{\beta}{2}\right]\right)
\end{aligned}
$$

It is obvious that the first three terms' approximate solutions (65)-(67) obtained using MDM are the same as the first four terms' (68)-(70) of the HPM.

ADM and HPM provide analytical solution in terms of an infinite power series (see (16) for ADM and (29)-(31) for HPM). The series consists of both positive and negative terms, although not in a regular alternating fashion. The ratio test was applied to the absolute values of the series coefficient. This provides a sufficient condition for convergence of the series for a space interval $\Delta X$ in the form:

$$
\lim _{m \rightarrow \infty}\left|\frac{a_{m+1}}{a_{m}}\right|<\frac{1}{\Delta X} .
$$

However, the approach in this study was to replace (71) with

$$
\lim _{m \rightarrow M}\left|\frac{a_{m+1}}{a_{m}}\right|<\frac{1}{\Delta X},
$$

where $M$ is a large constant. Figures 1, 2, and 3 show the behavior of the function $f(m)=\left|a_{m+1} / a_{m}\right|$ for increasing values of $m$. It is clear from these figures that the ratio $f(m)$ decays as $m$ increases, obviously indicating that the series is convergent.

Figures 4, 5, 6, and 7 show four snapshots in time of the tumour cell density, ECM density, and MDE concentration. The ECM profile shows clearly the degradation by the MDEs. As the MDEs degrade the ECM, the tumour cells invade via combination of diffusion and haptotaxis.

The tumour density distribution shows a small cluster of cells built up at the leading edge of the tumour due to haptotactic migration. As time evolves (Figures 5-7), this cluster of cells migrates further from the tumour main body and continues to invade the ECM at slower rate. 


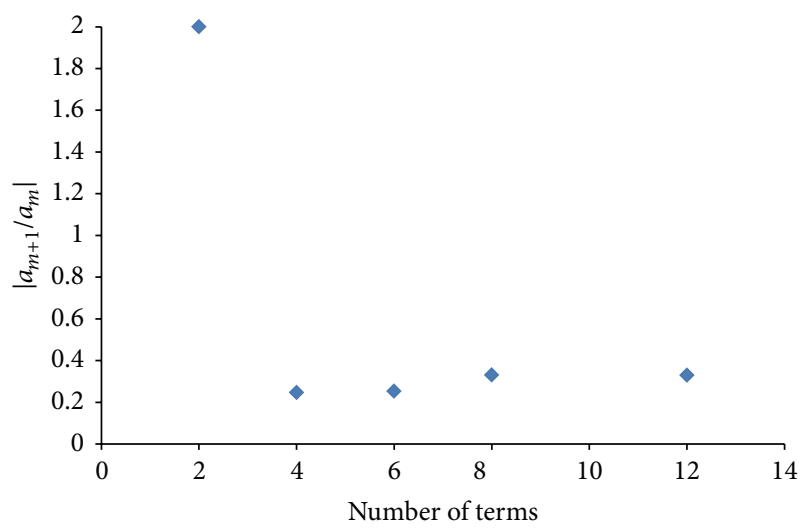

FIGURE 1: The ratio convergence test applied to the series coefficients (tumour) for MDM and HPM as a function of the number of terms in series.

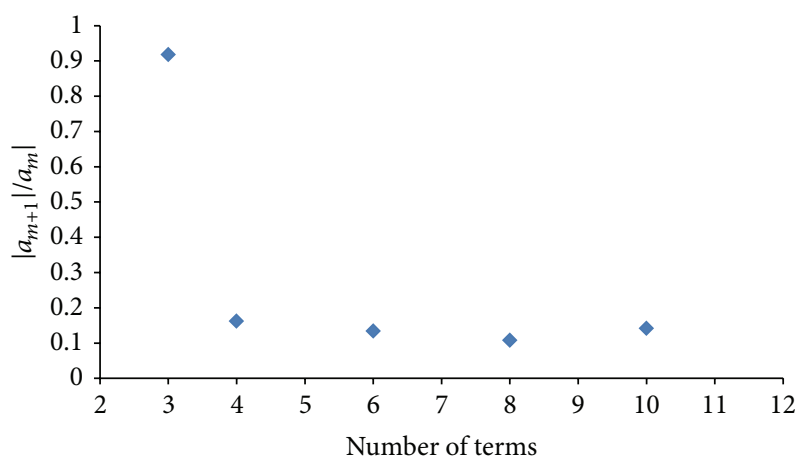

FIGURE 2: The ratio convergence test applied to the series coefficients (ECM) for MDM and HPM as a function of the number of terms in series.

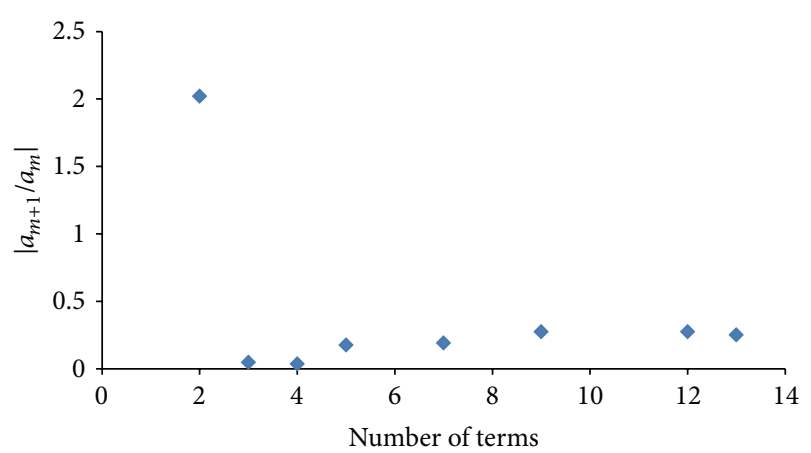

FIGURE 3: The ratio convergence test applied to the series coefficients (MDE) for MDM and HPM as a function of the number of terms in series.

\section{Conclusion}

In this paper, the modified decomposition method (MDM) and homotopy perturbation method (HPM) were used to obtain the solutions for the nonlinear model of tumour invasion and metastasis. Although the main difference between $\mathrm{MDM}$ and $\mathrm{ADM}$ is a slight variation in the definition of

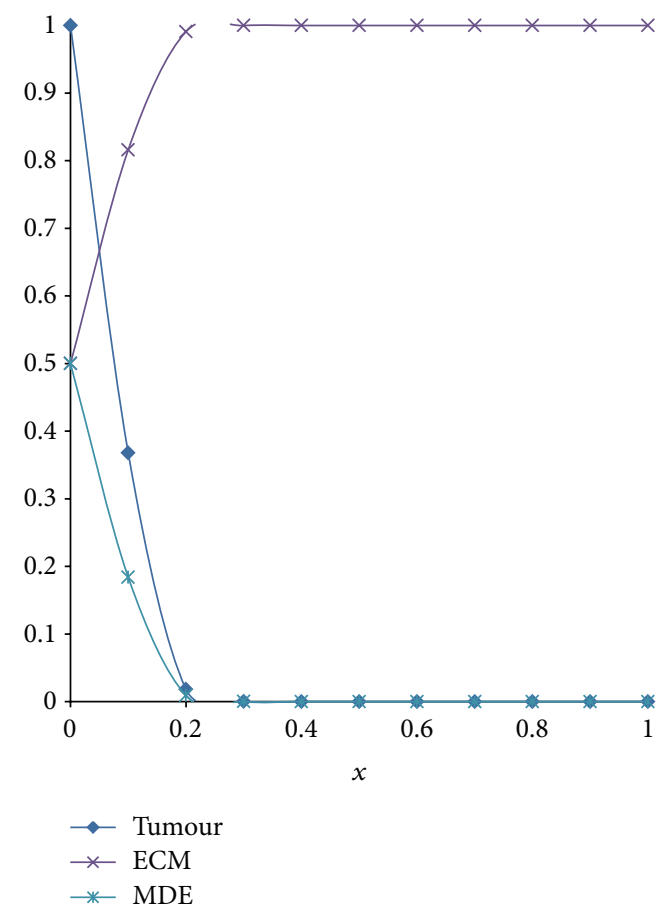

FIGURE 4: One-dimensional MDM and HPM solution of the system (3)-(5) with constant tumour cell diffusion showing the cell density, MDE concentration, and ECM density at $t=0$.

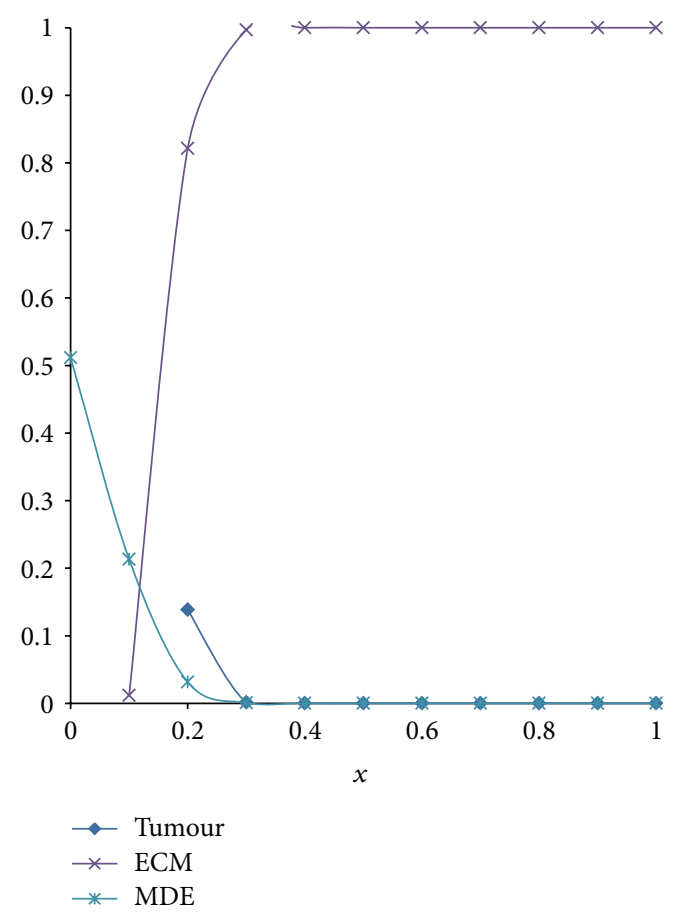

FIGURE 5: One-dimensional MDM and HPM solution of the system (3)-(5) with constant tumour cell diffusion showing the cell density, MDE concentration, and ECM density at $t=1$. 


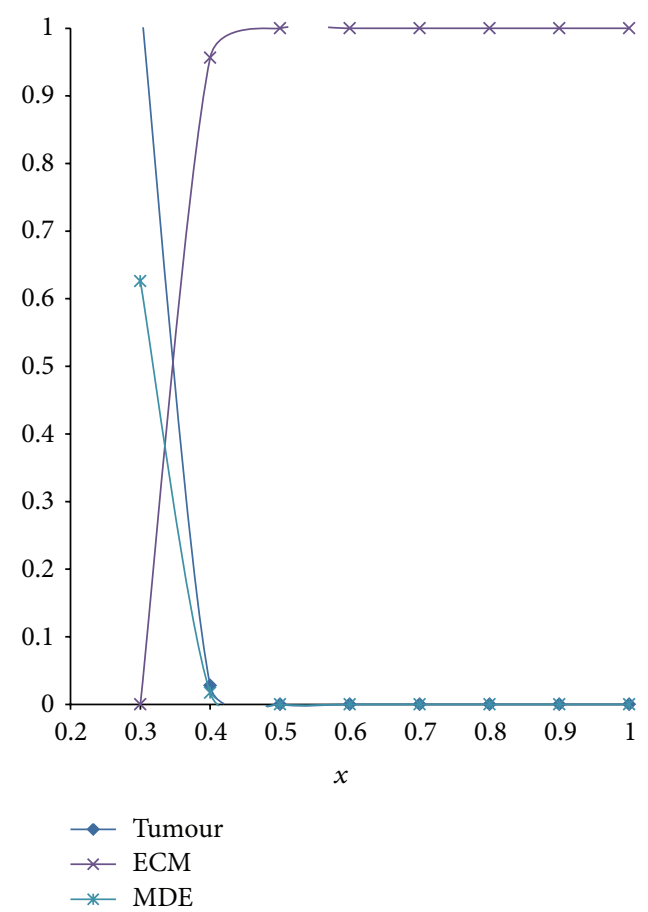

FIGURE 6: One-dimensional MDM and HPM solution of the system (3)-(5) with constant tumour cell diffusion showing the cell density, MDE concentration, and ECM density at $t=10$.

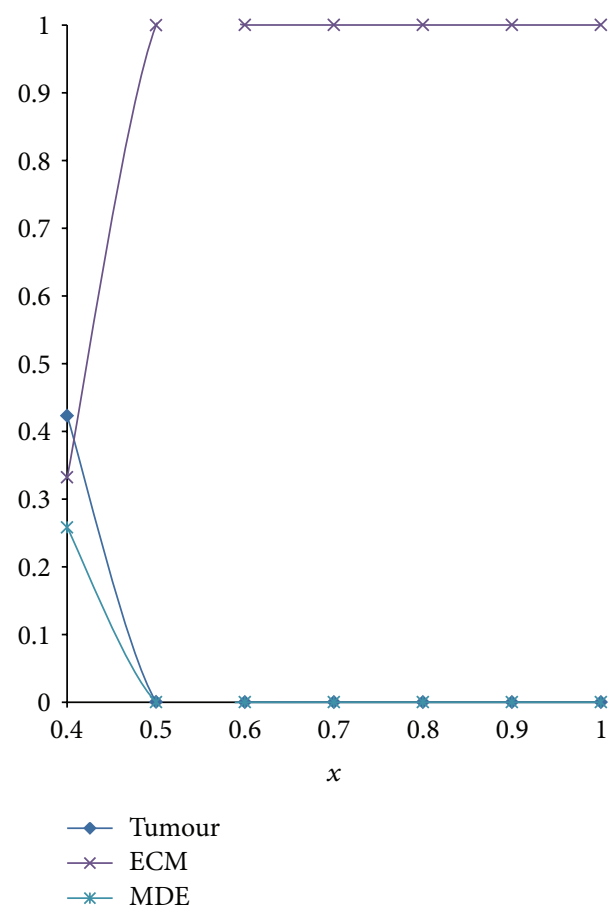

FIGURE 7: One-dimensional MDM and HPM solution of the system (3)-(5) with constant tumour cell diffusion showing the cell density, MDE concentration, and ECM density at $t=20$. the initial conditions, the modification demonstrates reliability and effectiveness in applying the present problem. This method thus eliminates the difficulties and massive computation work. Also it is shown that the obtained solution by MDM logically contains the solution obtained by HPM. The benefits of HPM with respect to MDM are HPM does not involve the Adomian polynomials which is a fundamental qualitative difference in analysis between HPM and MDM.

\section{Conflict of Interests}

The authors declare that there is no conflict of interests regarding the publication of this paper.

\section{Acknowledgments}

This work is financially supported by the PPP Grant (P0257/2007A) provided by University of Malaya. The authors gratefully acknowledge the financial support.

\section{References}

[1] M. A. J. Chaplain and A. M. Stuart, "A model mechanism for the chemotactic response of endothelial cells to tumour angiogenesis factor," IMA Journal of Mathematics Applied in Medicine and Biology, vol. 10, no. 3, pp. 149-168, 1993.

[2] M. A. J. Chaplain, "The mathematical modelling of tumour angiogenesis and invasion," Acta Biotheoretica, vol. 43, no. 4, pp. 387-402, 1995.

[3] A. R. A. Anderson and M. A. J. Chaplain, "Continuous and discrete mathematical models of tumor-induced angiogenesis," Bulletin of Mathematical Biology, vol. 60, no. 5, pp. 857-899, 1998.

[4] J. A. Sherratt and M. A. Nowak, "Oncogenes, Anti-Oncogenes and the immune response to cancer: a mathematical model," Proceedings of the Royal Society of London B, vol. 248, no. 1323, pp. 261-271, 1992.

[5] R. A. Gatenby and E. T. Gawlinski, "A reaction-diffusion model of cancer invasion," Cancer Research, vol. 56, no. 24, pp. 5745$5753,1996$.

[6] M. A. J. Chaplain, "Avascular growth, angiogenesis and vascular growth in solid tumours: the mathematical modelling of the stages of tumour development," Mathematical and Computer Modelling, vol. 23, no. 6, pp. 47-87, 1996.

[7] G. Adomian, "A review of the decomposition method and some recent results for nonlinear equations," Computers \& Mathematics with Applications, vol. 21, no. 5, pp. 101-127, 1991.

[8] J.-H. He, "Homotopy perturbation technique," Computer Methods in Applied Mechanics and Engineering, vol. 178, no. 3-4, pp. 257-262, 1999.

[9] I. Hashim, M. S. M. Noorani, R. Ahmad, S. A. Bakar, E. S. Ismail, and A. M. Zakaria, "Accuracy of the adomian decomposition method applied to the Lorenz system," Chaos, Solitons \& Fractals, vol. 28, no. 5, pp. 1149-1158, 2006.

[10] S. M. El-Sayed and M. R. Abdel-Aziz, "A comparison of Adomian's decomposition method and wavelet-Galerkin method for solving integro-differential equations," Applied Mathematics and Computation, vol. 136, no. 1, pp. 151-159, 2003. 
[11] A.-M. Wazwaz, "A reliable modification of Adomian decomposition method," Applied Mathematics and Computation, vol. 102, no. 1, pp. 77-86, 1999.

[12] A. R. A. Anderson, M. A. J. Chaplain, E. L. Newman, R. J. C. Steeele, and A. M. Thompson, "Mathematical modelling of tumour invasion and metastasis," Journal of Theoretical Medicine, vol. 2, no. 2, pp. 129-154, 2000.

[13] I. L. El-Kalla, "Convergence of the Adomian method applied to a class of nonlinear integral equations," Applied Mathematics Letters, vol. 21, no. 4, pp. 372-376, 2008.

[14] S. S. Behzadi and A. Yildirim, "A method to estimate the solution of weakly singular non-linear integro-differential equations by applying the Homotopy methods," International Journal of Industrial Mathematics, vol. 4, no. 1, pp. 41-51, 2012. 


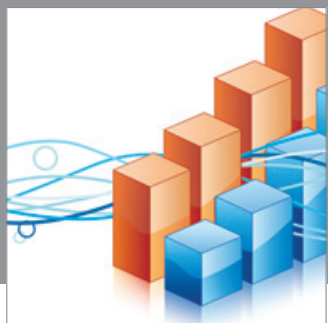

Advances in

Operations Research

mansans

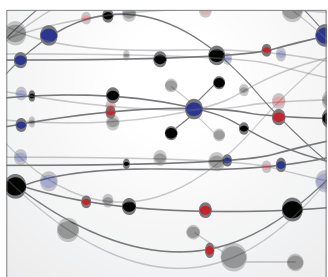

The Scientific World Journal
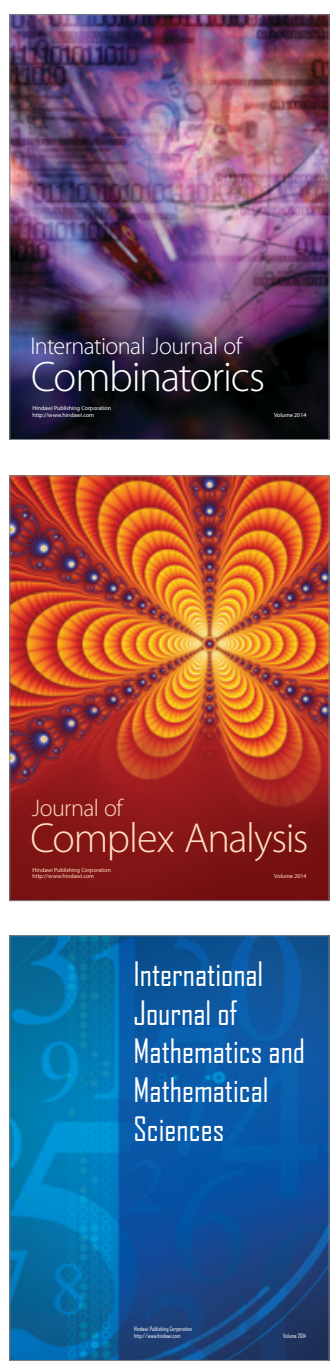
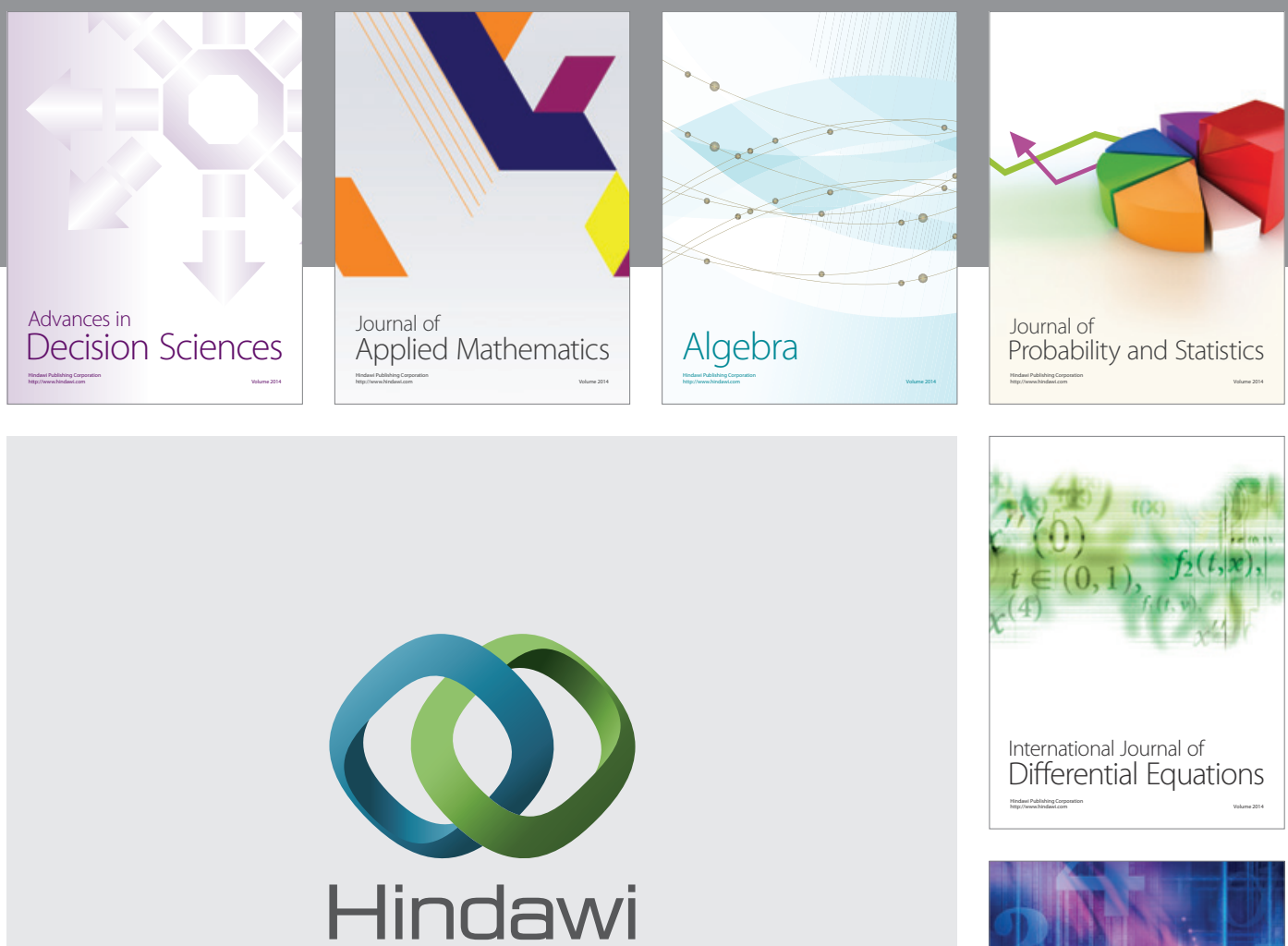

Submit your manuscripts at http://www.hindawi.com
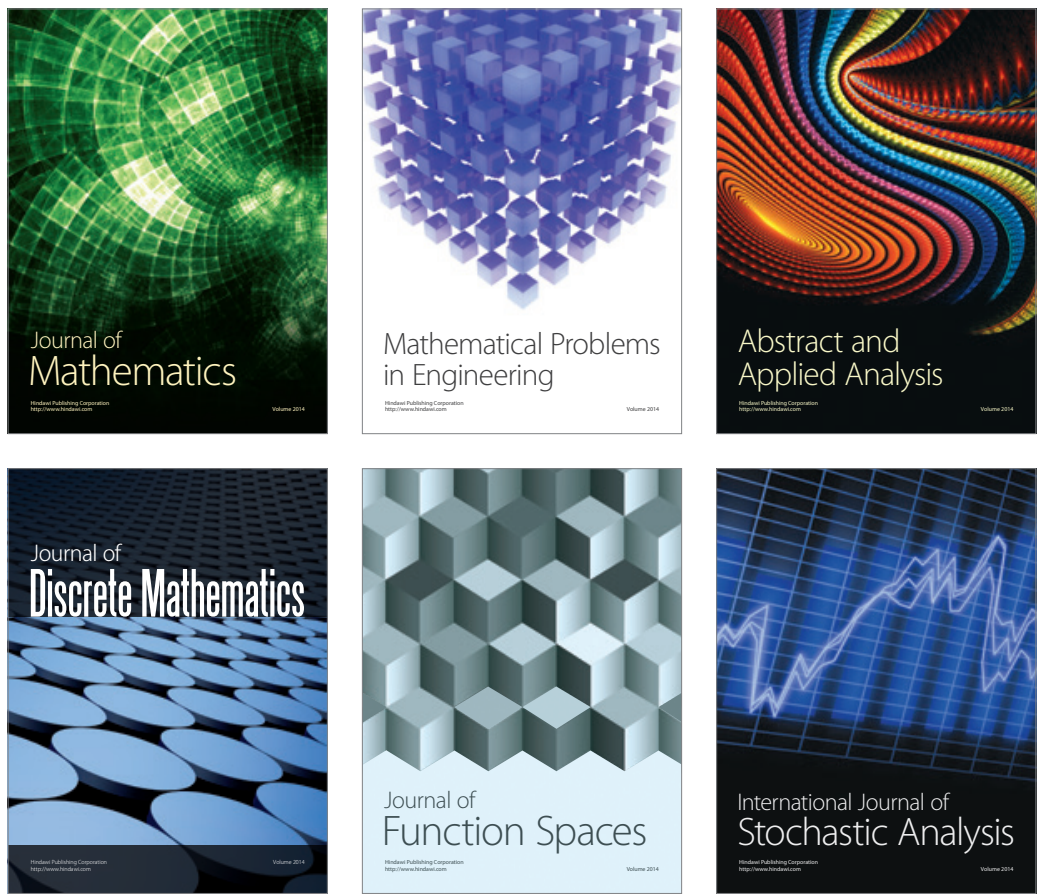

Journal of

Function Spaces

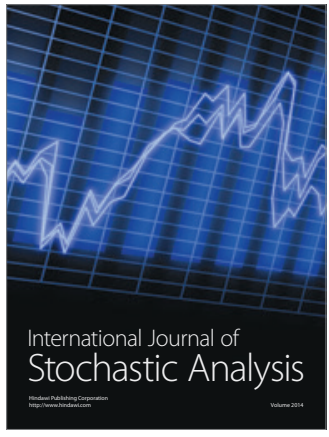

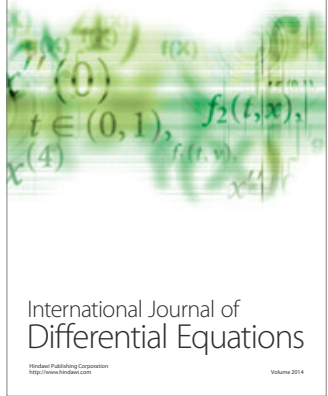
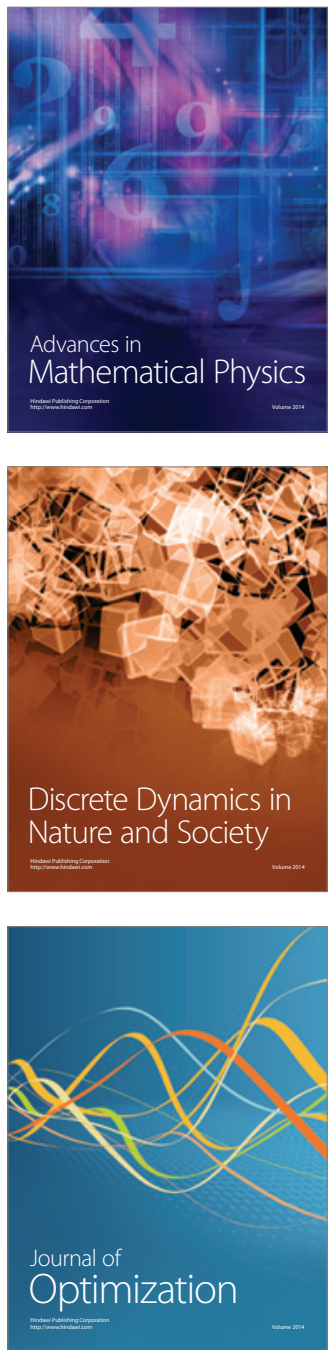